\title{
Interval dosing with the HDAC inhibitor vorinostat effectively reverses HIV latency
}

\author{
Nancie M. Archin, ${ }^{1,2}$ Jennifer L. Kirchherr, ${ }^{1}$ Julia A.M. Sung, ${ }^{1,2}$ Genevieve Clutton, ${ }^{1,3}$ Katherine Sholtis, ${ }^{1}$ Yinyan Xu, ${ }^{1}$ Brigitte Allard, ${ }^{1}$ \\ Erin Stuelke, ${ }^{1}$ Angela D. Kashuba, ${ }^{4}$ Joann D. Kuruc, ${ }^{1,2}$ Joseph Eron, ${ }^{1,2,5}$ Cynthia L. Gay, ${ }^{1,2}$ Nilu Goonetilleke, ${ }^{1,3}$ and David M. Margolis ${ }^{1,2,3,5}$ \\ 'University of North Carolina (UNC) HIV Cure Center, UNC Institute of Clobal Health and Infectious Diseases, ${ }^{2}$ Departments of Medicine and ${ }^{3}$ Microbiology and Immunology, UNC Chapel Hill School of \\ Medicine, ${ }^{4}$ Division of Pharmacotherapy and Experimental Therapeutics, UNC Eshelman School of Pharmacy, ${ }^{5}$ Department of Epidemiology, UNC Chapel Hill School of Public Health, University of North \\ Carolina at Chapel Hill, Chapel Hill, North Carolina, USA.
}

\begin{abstract}
BACKGROUND. The histone deacetylase (HDAC) inhibitor vorinostat (VOR) can increase HIV RNA expression in vivo within resting $\mathrm{CD4}^{+} \mathrm{T}$ cells of aviremic HIV+ individuals. However, while studies of VOR or other HDAC inhibitors have reported reversal of latency, none has demonstrated clearance of latent infection. We sought to identify the optimal dosing of VOR for effective serial reversal of HIV latency.
\end{abstract}

METHODS. In a study of 16 HIV-infected, aviremic individuals, we measured resting CD4 ${ }^{+} \mathrm{T}$ cell-associated HIV RNA ex vivo and in vivo following a single exposure to VOR, and then in vivo after a pair of doses separated by 48 or 72 hours, and finally following a series of 10 doses given at 72 -hour intervals.

RESULTS. Serial VOR exposures separated by 72 hours most often resulted in an increase in cell-associated HIV RNA within circulating resting $\mathrm{CD}^{+} \mathrm{T}$ cells. VOR was well tolerated by all participants. However, despite serial reversal of latency over 1 month of VOR dosing, we did not observe a measurable decrease $\left(>0.3 \log _{10}\right)$ in the frequency of latent infection within resting CD4 ${ }^{+}$cells.

CONCLUSIONS. These findings outline parameters for the experimental use of VOR to clear latent infection. Latency reversal can be achieved by VOR safely and repeatedly, but effective depletion of persistent HIV infection will require additional advances. In addition to improvements in latency reversal, these advances may include the sustained induction of potent antiviral immune responses capable of recognizing and clearing the rare cells in which HIV latency has been reversed.

TRIAL REGISTRATION. Clinicaltrials.gov NCT01319383.

FUNDING. NIH grants U01 AI095052, AI50410, and P30 CA016086 and National Center for Advancing Translational Sciences grant KL2 TR001109.

\section{Introduction}

Current approaches to achieve a cure for HIV infection include the administration of latency-reversing agents (LRAs) to induce latently HIV-infected cells to reexpress virions or viral antigens, in concert with immunotherapies to clear these newly vulnerable targets. Sustained or repeated disruption of latency by LRAs is paramount for the success of this approach. Therefore, defining effective LRA dosing regimens that lead to repeated HIV RNA expression and HIV antigen presentation is an important first step toward targeting persistent HIV infection. To date, histone deacetylase (HDAC) inhibitors are the most well-characterized class of LRAs studied in humans as a modality to disrupt HIV latency in vivo.

Conflict of interest: J. Eron reports consultancy for Merck, Gilead, and Janssen, and research funding from Gilead and Janssen. C.L. Gay reports research funding from Cilead, Viiv, and Janssen. A.D. Kashuba and D.M. Margolis report consultancy for Merck D.M. Margolis holds common stock in Gilead.

Submitted: January 6, 2017; Accepted: June 1, 2017.

Reference information: J Clin Invest. 2017;127(8):3126-3135.

https://doi.org/10.1172/JCI92684.
HDACs are a class of enzymes that repress transcription primarily through their ability to covalently modify the lysine tail of core histones of nucleosomes through deacetylation, but can also exert influences on cellular activities that are independent of transcriptional repression (reviewed in ref. 1). Deacetylation of lysine residues on histone tails decreases the access of transcription factors to the DNA and recruits histone-modifying and chromatin complexes that result in transcriptional repression. HDAC inhibitors generally function by blocking the catalytic domain of HDACs and thereby interfering with their deacetylase activity. Of the 4 known classes of HDACs (class I, class IIa, class IIb, class III, and class IV), the class I HDACs are reported to be important in HIV transcriptional repression (2). Most HDAC inhibitors investigated in the clinic as anti-HIV latency therapeutics are selective for class I HDACs or have broad activity against HDACs $(1,3)$.

We first reported that a single 400-mg dose of vorinostat (VOR) given to HIV-infected but stably aviremic individuals on antiretroviral therapy (ART) can increase HIV RNA in resting $\mathrm{CD} 4^{+} \mathrm{T}$ cells, demonstrating the reversibility of latency in humans (4), a finding corroborated in studies of total $\mathrm{CD} 4^{+} \mathrm{T}$ cells using 


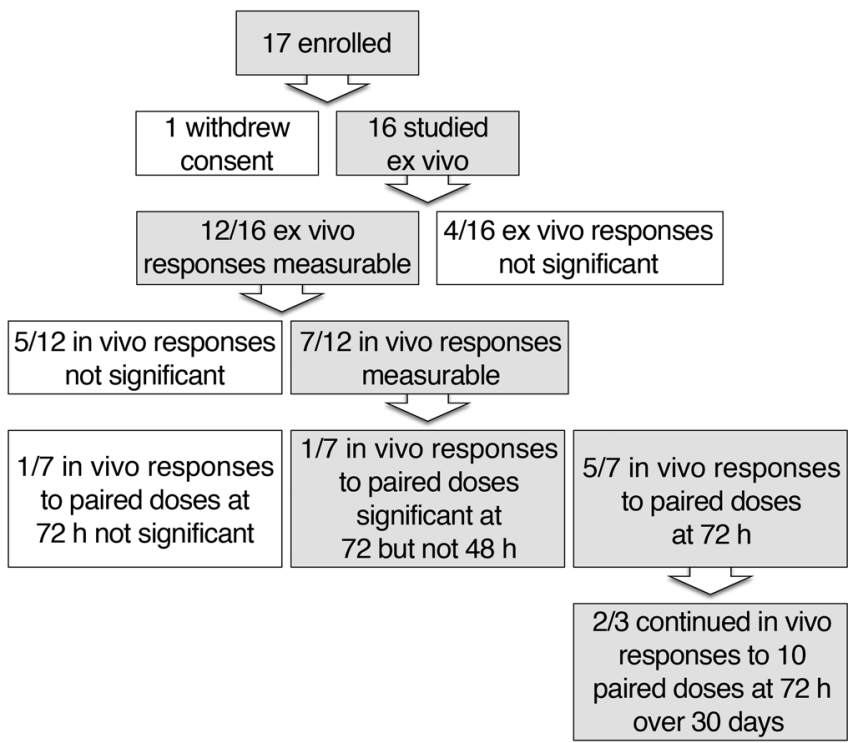

Figure 1. Study participant allocation and outcome.

VOR, the pan-HDAC inhibitor panobinostat, or the class I inhibitor romidepsin (5-7). In a subsequent study, 5 participants from our initial study elected to enter into a multi-dose arm, in which VOR was administered daily for 3 days, followed by a 4 -day rest period, for 6 cycles. Using this regimen, we observed a waning of the ability of VOR to consistently and significantly induce RNA expression in all patients (8).

To examine this effect, a population pharmacokinetic/pharmacodynamic model was built from all available daily VOR dosing and histone acetylation data for the 5 participants, using nonlinear mixed-effects modeling with NONMEM 7.2 (9). This model was used to construct exploratory exposure-effect relationships. It showed a clockwise hysteresis loop suggesting that the relationship between VOR concentration and HIV LRA activity is not a simple, direct effect. This could result in part from HDAC inhibitor effects on host cellular function (10).

Consistent with the potential for complex interactions between the cellular effects of HDAC inhibition and the expression of latent HIV, several studies reported that exposure to HDAC inhibitors is associated with a subsequent downregulation of positive regulators of transcription, such as histone acetyl transferases (HATs) $(11,12)$. Furthermore, HDAC inhibition can lead to an increase in trimethylation of lysine 27 on histone 3 (H3K27me3) at transcriptional start sites (12). H3K27me3 is a marker associated with transcriptional repression. These reports underscore the importance of understanding the kinetics of the HIV transcriptional response following serial HDAC inhibitor exposure, to maximize HIV LRA activity, and suggest that the effects of such exposure over time may be complex and will require detailed study.

A variety of assays have been used in translational studies to assess the reversal of HIV latency in vivo and have most often involved PCR-based amplification of cell-associated HIV RNAs in a variety of cell populations $(13,14)$. In order to focus our study on the disruption of proviral latency in resting $\mathrm{CD} 4^{+} \mathrm{T}$ cells in the most rigorous way, we elected to assess large numbers of circulating resting $\mathrm{CD} 4^{+} \mathrm{T}$ cells obtained via apheresis. Changes in HIV RNA levels within the resting $\mathrm{CD}^{+}{ }^{+} \mathrm{T}$ cell pool are most likely to represent the true reversal of latency and recent upregulation of HIV proviral expression, rather than HIV expression in the "active reservoir" of HIV-RNA-expressing cells found in circulating total $\mathrm{CD}^{+}{ }^{+} \mathrm{T}$ cell pools (15). Further, the measurement of resting $\mathrm{CD}^{+}{ }^{+} \mathrm{T}$ cell-associated HIV RNA (rca-HIV RNA) in 36 replicate pools of 1 million resting $\mathrm{CD} 4^{+} \mathrm{T}$ cells ensures a robust and precise assessment of changes in HIV RNA expression (4). Thus, we used this approach to explore the optimal VOR dosing interval that would result in consistent and sustained reversal of HIV latency. Further, as an antiviral immune response is thought to be required to clear persistent HIV infection following LRA activity, we measured immune function following prolonged exposure to VOR in vivo.

\section{Results}

In vitro modeling of effective VOR dosing. Prior to executing a clinical study (Figure 1), we used an in vitro approach to explore the dosing schedule that might lead to consistent and sustained expression of rca-HIV RNA after exposure of resting $\mathrm{CD}^{+} \mathrm{T}$ cells to VOR. To do this, we took advantage of previously reported in vivo VOR pharmacokinetics showing maximum exposure to VOR between 3 and 6 hours after a 400-mg dose, followed by rapid clearance (4).

We studied resting $\mathrm{CD}^{+} \mathrm{T}$ cells isolated from aviremic patients, exposing these cells to $335 \mathrm{nM}$ VOR ex vivo for 6 hours. This VOR exposure models the effects achieved after a 400-mg oral dose (4). Cells were then washed and placed back into the culture. To mimic daily, every-other-day, and every-3-day exposure to VOR, the cells were then given a second pulse of VOR after an 18-, 42-, or 66-hour drug-free interval in culture (Figure 2A). As expected, we observed a significant increase in cell-associated HIV RNA after the initial pulse of VOR (Figure 2B). Consistent with our previous in vivo study (8), we observed a consistent dampening of the response to VOR when cells received a second VOR exposure 18 hours after the previous exposure was terminated (Figure 2C). Importantly, if the cells were exposed to the second dose of VOR after resting for either 42 or 66 hours, we again observed a significant increase in cell-associated HIV RNA (Figure 2, D and E), suggesting that more than 18 hours without HDAC inhibitor exposure may be necessary to allow the HIV promoter and/or infected cell to "reset" and respond to subsequent doses of VOR.

Clinical outcome of single, paired, and multiple doses of VOR. Given these in vitro pilot data, 16 HIV-infected, aviremic, ART-treated participants were enrolled in a clinical study with the objective of determining the optimal in vivo VOR dosing schedule necessary for effective serial disruption of the $\mathrm{CD} 4^{+} \mathrm{T}$ cell reservoir. VOR dosing of $400 \mathrm{mg}$ was derived from oncology studies, with the goal of achieving maximal drug exposure with negligible toxicities, as in our original studies (4). An initial leukapheresis evaluation was performed to obtain resting $\mathrm{CD} 4^{+} \mathrm{T}$ cells for quantitation of baseline resting $\mathrm{CD} 4^{+} \mathrm{T}$ cell infection (RCI) and rca-HIV RNA and validation of a measurable response to an ex vivo dose of VOR, as previously described (8).

Given the theoretical risks of VOR, the fact that it is unlikely that participants would derive clinical benefit from this study, the limitations in the number of leukapheresis evaluations that could 

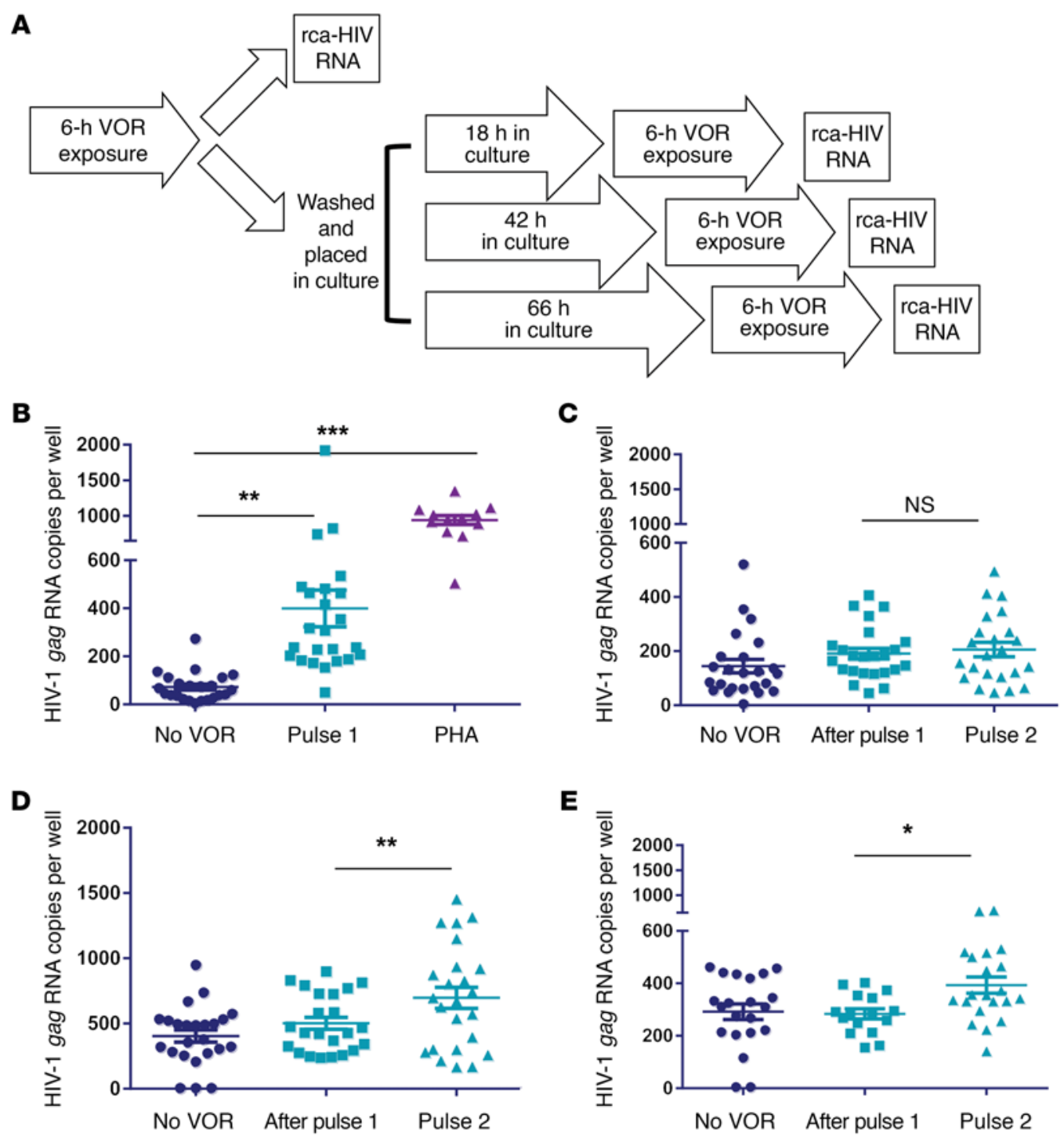

Figure 2. Ex vivo modeling of serial VOR exposures. (A) Schematic of ex vivo dosing experiment. Resting CD4 ${ }^{+} \mathrm{T}$ cells from aviremic participants were exposed to media or $335 \mathrm{nM}$ VOR (or PHA as a comparative control) for 6 hours, rca-HIV RNA was measured in an aliquot (B), and the remaining cells were washed and placed in culture. Cells then received no VOR or a second 6-hour pulse of VOR at 18 hours, 42 hours, or 66 hours following the first pulse to model daily, every-2-day, or every-3-day dosing regimens. rca-HIV RNA was measured in cells that had never been exposed to VOR (circles), were pulsed once (squares), or pulsed twice (triangles) at 24 hours (C), 48 hours (D), and 72 hours (E). Each data point represents 1 million resting CD4 ${ }^{+} T$ cells. $n=3$ independent experiments. Data represent the mean $\pm S D$. ${ }^{*} P<0.05$, ${ }^{* *} P<0.005$, and ${ }^{* * *} P<0.0001$, by Mann-Whitney $U$ test.

be performed to precisely measure rca-HIV RNA and resting cell infection, and to avoid fruitless VOR exposure for participants in whom an endpoint was not quantifiable, continued study evaluation was restricted to those who showed a significant increase in rca-HIV RNA after each step of the protocol (Figure 1 and Table 1). We first confirmed that a significant increase in rca-HIV RNA could be measured upon ex vivo exposure.

In those with a measurable ex vivo induction of rca-HIV RNA (12 of 16), a single dose of VOR was administered, and rcaHIV RNA response, safety, and tolerability were ascertained as described previously (4). Subsequently, qualified participants with a measurable induction of rca-HIV RNA following a single dose of VOR $(n=7)$ received a paired dose of VOR. For 1 participant, the doses were administered at a 48-hour interval and then later at a 72-hour interval. Six subsequent participants were administered paired doses at seventy-two hours. Three participants with a significant increase in rca-HIV RNA after the second dose in the pair (third dose total) then agreed to receive ten doses of VOR, given every seventy-two hours. The frequency of rca-HIV RNA and RCI frequency was evaluated via leukapheresis after the tenth serial dose of VOR ( $13^{\text {th }}$ dose total).

The dosing regimen was well tolerated by all patients, with some mild, transient gastrointestinal symptoms reported, none of which approached grade I, according to the Division of AIDS (DAIDS) Toxicity Table for Grading Adverse Events. All 3 participants who advanced to the month-long serial dosing phase showed a $15 \%-35 \%$ decline in platelet counts that recovered to baseline levels when evaluated 4-8 weeks after dosing com- 
Table 1. Baseline characteristics of the study participants

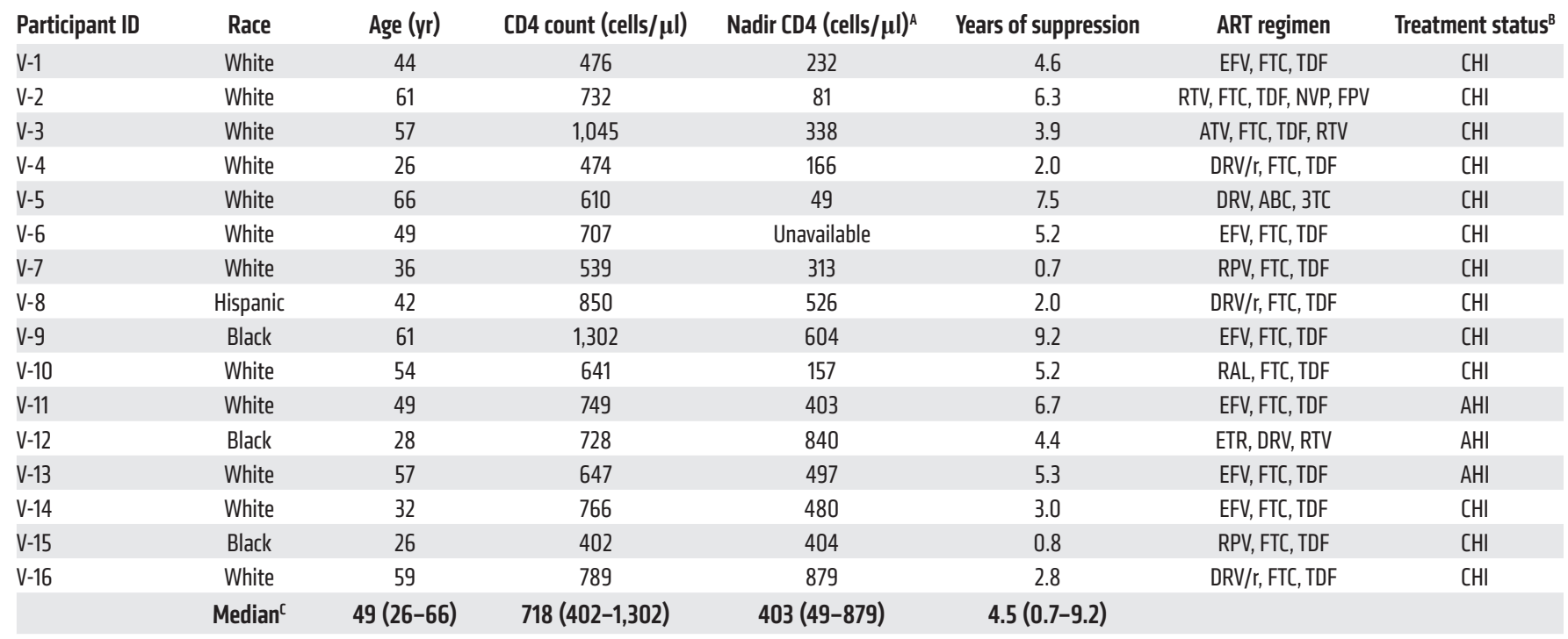

ALowest recorded CD4 cell count in available clinical records. ${ }^{B} \mathrm{CHI}$, treated during chronic HIV infection; AHI, acute HIV infection defined as detectable HIV-1 RNA in an antibody-negative or indeterminate sample and initiated ART within 45 days of the estimated date of infection, which was calculated as 14 days prior to the onset of symptoms consistent with acute retroviral syndrome. ${ }^{\mathrm{C}} \mathrm{All}$ participants were men; the median plus the range are shown. EFV, efavirenz; FTC, emtricitabine; TDF, tenofovir disoproxil fumarate; RTV, ritonavir; NVP, nevirapine; FPV, fosamprenavir; ATV, atazanavir; DRV/r, darunavir/ ritonavir; ABC, abacavir; 3TC, lamivudine; RAL, raltegravir; ETR, etravirine; RPV, rilpivirine.

pletion. This transient thrombocytopenia never reached the threshold to qualify as a grade I adverse event. No other clinically significant events or drug-related toxicities were observed.

In vivo administration of VOR every 72 hours leads to a sustained increase in rca-HIV RNA. Participants with an increase in measurable HIV RNA after ex vivo exposure to VOR were administered a single dose of VOR. Among the 16 participants enrolled, we were able to measure a significant increase in HIV RNA after ex vivo exposure to VOR in the resting $\mathrm{CD}^{+}$cells of $12(75 \%)$ individuals (Figure 3$)$. Among the nonresponders, in 1 (V-13), the level of rcaHIV RNA in the cells before exposure was detectable but not quantifiable, and we were not able to measure a response to VOR (Supplemental Figure 1; supplemental material available online with this article; https:/doi. org/10.1172/JCI92684DS1). In the cells of 1 participant (V-16) with a high pre-exposure rca-HIV RNA level, we detected a marginal increase in RNA, even when the cells were stimulated with mitogen (Supplemental Figure 1). In 2 other participants (V-14 and $\mathrm{V}-15)$, we detected an increase in HIV RNA that did not achieve statistical significance (Supplemental Figure 1).

A single in vivo dose of VOR was then administered to the 12 individuals mentioned above. We observed a measurable and significant increase in cell-associated RNA in resting $\mathrm{CD}^{+} \mathrm{T}$ cells isolated from 7 of the 12 participants (Figure 4 ) and no increase in 4 participants (Supplemental Figure 2), although baseline and post-VOR dose levels measured in 1 participant (V-12) were very low. In 1 participant (V-9), the baseline rca-HIV RNA was unexpectedly higher than that measured following dosing (Supplemental Figure 2). However, it was retrospectively confirmed that this patient had an episode of transient viremia $(150 \mathrm{c} / \mathrm{ml})$ immediately prior to commencement of the study, potentially confounding the ascertainment of a response to VOR.

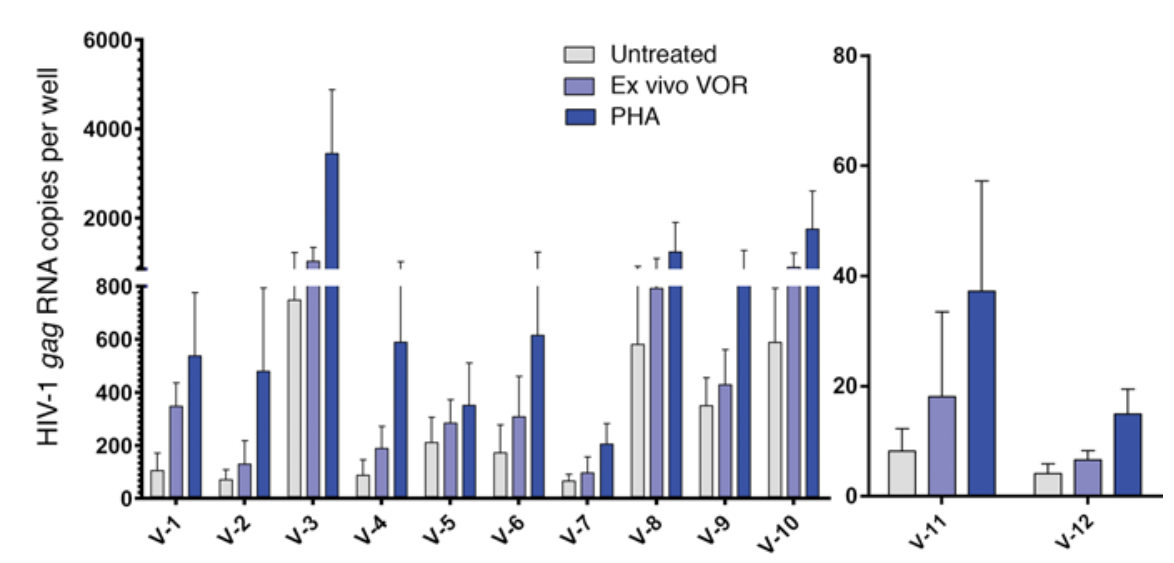

Figure 3. A 6-hour exposure to VOR ex vivo increased HIV gag RNA in resting CD4 ${ }^{+} \mathrm{T}$ cells isolated from aviremic donors. Pools of 24 million resting $\mathrm{CD} 4^{+} \mathrm{T}$ cells isolated from aviremic donors were exposed to $335 \mathrm{nM}$ VOR or mitogen (PHA and $60 \mathrm{U} / \mathrm{ml} \mathrm{IL-2)} \mathrm{for} 6$ hours, and rca-HIV RNA levels were measured. Data represent the mean $\pm \mathrm{SD}$. $P<0.05$ to $P<0.0001$ (Mann-Whitney $U$ test) for all comparisons between untreated cells and ex vivo VOR-treated cells. 


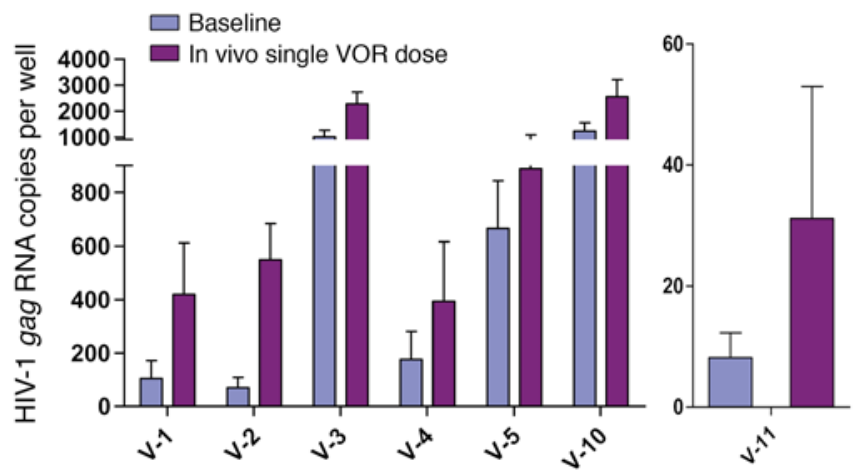

Figure 4. In vivo administration of a single 400-mg dose of VOR led to a significant increase in rca-HIV RNA in the resting $\mathrm{CD4}^{+} \mathrm{T}$ cells of aviremic participants. Data represent the mean \pm SD. $P<0.0001$ (Mann-Whitney $U$ test) for all comparisons between baseline and VOR treatment.

Given the in vitro data suggesting that daily drug exposure would blunt the viral response to serial VOR exposure, a conclusion also suggested by the results of our previous clinical trial (8), all participants with a significant increase in rca-HIV RNA after a single dose of VOR $(n=7)$ received paired doses at either a 48-hour or 72-hour interval. Patient V-11 was given a paired dose at a 48-hour interval, and as shown in Figure 5A, the increase in HIV RNA did not achieve statistical significance. This participant later received 2 doses of VOR at a 72-hour interval, and we detected a significant increase in rca-HIV RNA in resting CD $4^{+} \mathrm{T}$ cells (Figure $5 \mathrm{~A}$ ). Following this observation, and given anecdotal concerns that prolonged alternate-day dosing of VOR might be more difficult to tolerate, all 6 additional participants received VOR doses at the 72-hour interval. We observed a significant increase in rcaHIV RNA in 5 of these 6 individuals (Figure 5B). Sparse pharmacokinetic sampling of VOR levels near the predicted peak of exposure was similar to sampling levels in prior studies (data not shown) (4, 8). The lack of an increase in rca-HIV RNA in participant V-1 had no obvious explanation.

Strategies seeking to eradicate HIV infection are likely to require multiple cycles of LRA exposure to induce a sufficient display of viral antigen to allow the immune system to identify and clear these previously unrecognizable infected cells. To that end, 3 of the participants who showed a significant increase in rca-HIV RNA after receiving a paired dose of VOR 72 hours apart elected to receive 10 consecutive doses of VOR at this interval to determine whether HIV RNA expression could be sustained over a 1-month period of pulsatile VOR therapy. As shown in Figure 6, a significant, sustained increase in HIV RNA was detected in 2 of the 3 participants. Of note, participant $\mathrm{V}-5$, who did not show a continued significant induction of rca-HIV RNA, reported difficulty with adherence to the 72-hour dosing schedule, deviating from the schedule and taking 3 of the 10 doses at shorter intervals. Whether this nonadherence to protocol contributed to the lack of a measurable rca-HIV RNA response cannot be determined. Importantly, as in previous studies,we used a rigorous approach to measure rca-RNA, taking steps to minimize RNA degradation and ensuring that the increase in rca-HIV RNA measured after all VOR dosing was not due to baseline RNA variation over time (Supplemental Figure 3).
Finally, we evaluated the frequency of the replication-competent latent $\mathrm{HIV}$ within the resting $\mathrm{CD}^{+} \mathrm{T}$ cell reservoir by performing a quantitative viral outgrowth assay (QVOA) at baseline and following paired and multiple VOR doses. Like other studies of latency reversal $(4-8,16,17)$, we did not observe convincing evidence of a significant reduction $\left(>0.3 \log _{10}\right)$ in the frequency of virus recovered in the QVOA (Supplemental Table 1). Not surprisingly, we also did not observe any changes in the levels of cell-associated HIV DNA following in vivo administration of VOR (data not shown).

Multiple doses of VOR minimally impact immune effector cells in vivo. In light of these findings, future studies must seek to combine LRAs with modalities to augment the antiviral immune response to achieve clearance of persistent HIV infection. Therefore, we sought to demonstrate that the administration of VOR does not impede the action of effector cell populations critical for clearance of antigen-expressing cells. We therefore compared the ability of $\mathrm{CD}^{+} \mathrm{T}$ cells isolated from participants before, during, and after the receipt of 10 doses of VOR to proliferate following stimulation with HIV peptide pools. As shown in Figure 7A, we did not observe a significant change from baseline in the ability of $\mathrm{CD}^{+} \mathrm{T}$ cells to proliferate in the presence of a Flu/EBV peptide pool (FEC), an HIV Gag/Nef pool (CTLA), or an HIV non-Gag/Nef peptide pool (CTLB). Further, in a viral inhibition assay, the antiviral capacity of $C D 8^{+} \mathrm{T}$ cells when challenged with autologous targets infected with autologous reservoir virus $(18,19)$ tested at effector-to-target $(\mathrm{E} / \mathrm{T})$ ratios of 1:10 and 1:1 was not significantly altered following 10 doses of VOR. We observed no reduction in antiviral activity in the 3 participants, as analyzed by 1-way ANOVA with post-test correction for multiple comparisons (Figure 7B). Finally, during the course of the study, we observed a transient but marginal decline in absolute lymphocyte counts for some participants (Supplemental Table 2) that was resolved within weeks and was clinically insignificant.

\section{Discussion}

The ability to create a durable window of vulnerability within the latent reservoir of HIV infection via the repeated induction of viral antigen expression, allowing the clearance of persistent infection over a clinically tractable period of time, is paramount to developing strategies to eradicate HIV infection. Therefore, defining an effective HDAC inhibitor dosing regimen that can lead to HIV RNA and subsequent HIV antigen expression is an important first step toward this goal. We conducted a stepwise pilot study to determine the proper dosing interval for VOR, studying 48- and 72-hour intervals.

However, the effort to develop therapies that might clear HIV infection is relatively new, and the tools and assays currently at hand have limitations. The demonstration of a disruption of HIV latency, as measured by increased levels of HIV RNA within resting $\mathrm{CD}^{+} \mathrm{T}$ cell populations immediately following a single dose of VOR (4), was followed by the failure to uniformly observe latency reversal upon serial dosing of VOR given every 24 hours (8). Although the biomarker of the HDAC inhibitor effect, total cellular histone acetylation, returns to near-baseline levels within 24 hours of a single VOR dose (4), evidence suggests that the cellular effects of HDAC inhibitor exposure may be more durable (10, 11, 20-23). 
A

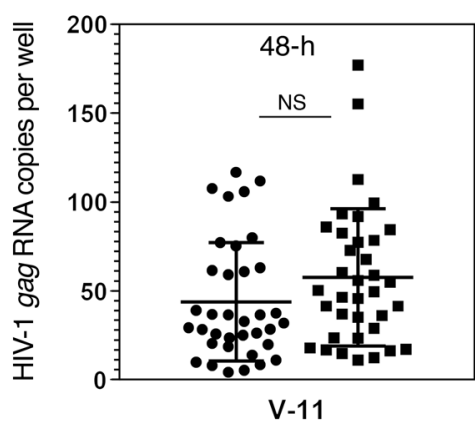

B
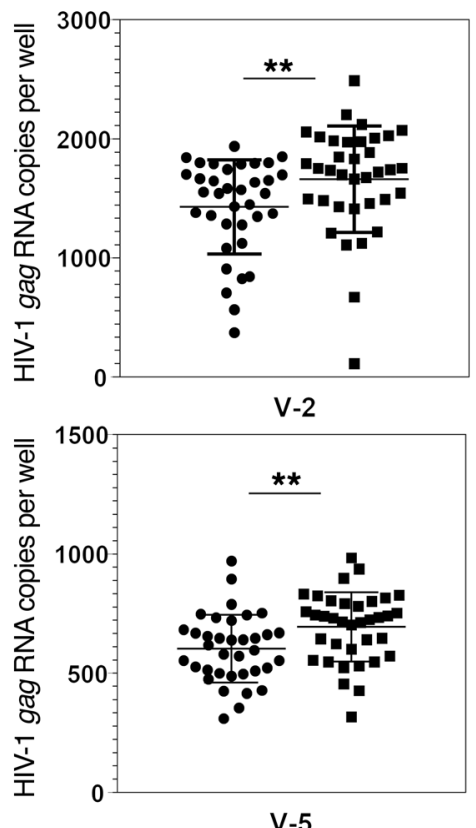

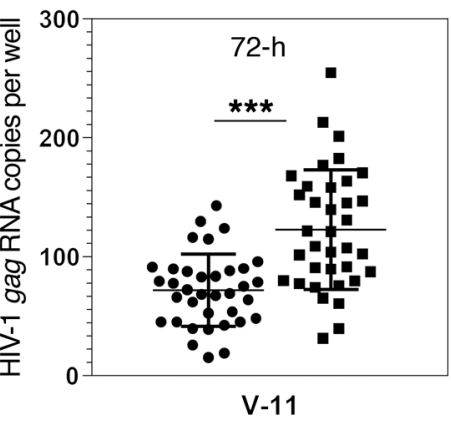

- Baseline

- In vivo paired dose
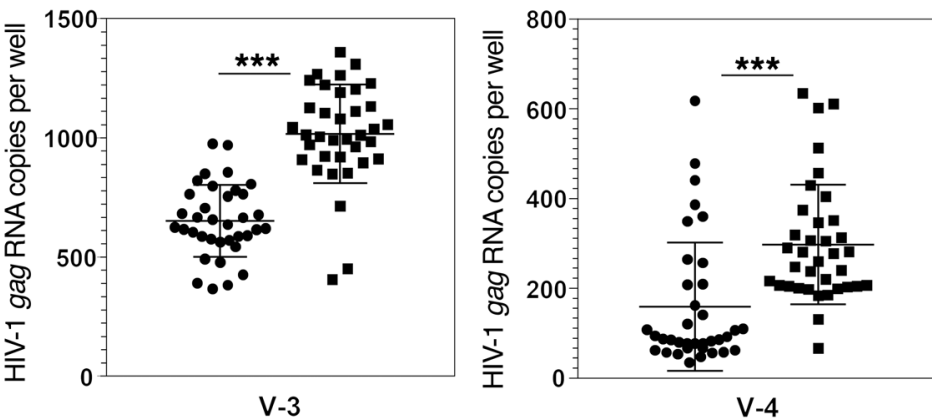

Baseline

In vivo paired dose $(72-h)$
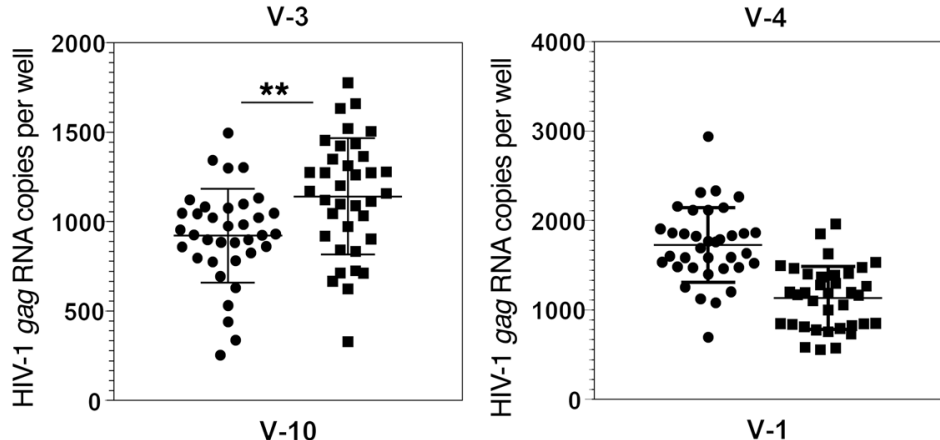

Figure 5. Two sequential doses of VOR given every $\mathbf{7 2}$ hours, but not 48 hours, resulted in a sustained increase in rca-HIV RNA in participants. (A) A 48-hour interval between sequential doses for 1 participant resulted in an increase in rca-HIV RNA levels that only became statistically significant when the interval between doses was increased to 72 hours. (B) A significant increase in rca-HIV RNA levels was observed in 5 of 6 additional participants at the 72-hour interval. Data represent the mean $\pm \mathrm{SD}$. ${ }^{* *} P<0.01$ and ${ }^{* *} P<0.0001$, by Mann-Whitney $U$ test. Baseline rca-HIV RNA levels are from samples collected at screening, either before the first VOR dose (V-3, V-4, V-5, and V-10), or from a second baseline sample collected prior to the paired dosing for participants with more than 1 year of elapsed time from administration of the first dose $(\mathrm{V}-1, \mathrm{~V}-2$, and $\mathrm{V}-11)$.

In studies that have assessed HIV RNA within total populations of circulating $\mathrm{CD}^{+}$T cells, 14 daily doses of VOR (5), panobinostat given 3 times per week on alternating weeks (6), and once-weekly administration of romidepsin (7) have suggested the occurrence of serial latency reversals, although the doseresponse effects were not consistent in all studies. Episodic viremia has been observed in some studies following HDAC inhibitor exposure (7), although it has not been shown that these episodes emanate from cells that were formerly latently infected.

Given the strong and uniform evidence in all prior studies that the initial exposure to the HDAC inhibitor disrupts HIV latency, and given the need to ascertain whether latency is serially reversed in the most important reservoir of persistent infection resting $\mathrm{CD}^{+}{ }^{+} \mathrm{T}$ cells, this study focused on measurements of rcaHIV RNA following multiple VOR doses.

To accomplish this, we followed a systematic protocol in which subsequent dosing was directed by the rca-HIV RNA response of each participant in a stepwise fashion. Given the demanding and resource-intensive nature of these evaluations, the number of participants evaluated is thus far limited, but these initial findings provide useful guidance for the design of future studies seeking to disrupt latency and clear persistent HIV infection. VOR given in this setting was very well tolerated, with only mild and reversible declines in platelet counts seen during 1 month of VOR exposure and no significant gastrointestinal toxicities.

In 6 of 7 participants who received VOR doses at 72-hour intervals, we observed a significant increase in rca-HIV RNA levels within circulating resting $\mathrm{CD} 4^{+} \mathrm{T}$ cells (Figure 5). Three of these 7 participants then received serial doses of VOR every 72 hours for 1 month, and significant increases in rca-HIV RNA levels within circulating resting $\mathrm{CD} 4^{+} \mathrm{T}$ cells $(P<0.01$ in three and $P<0.0001$ in three; Figure 5) were still observed following the tenth dose of VOR in 2 of the 3 participants. This substantial exposure to VOR was well tolerated, and an assessment of 


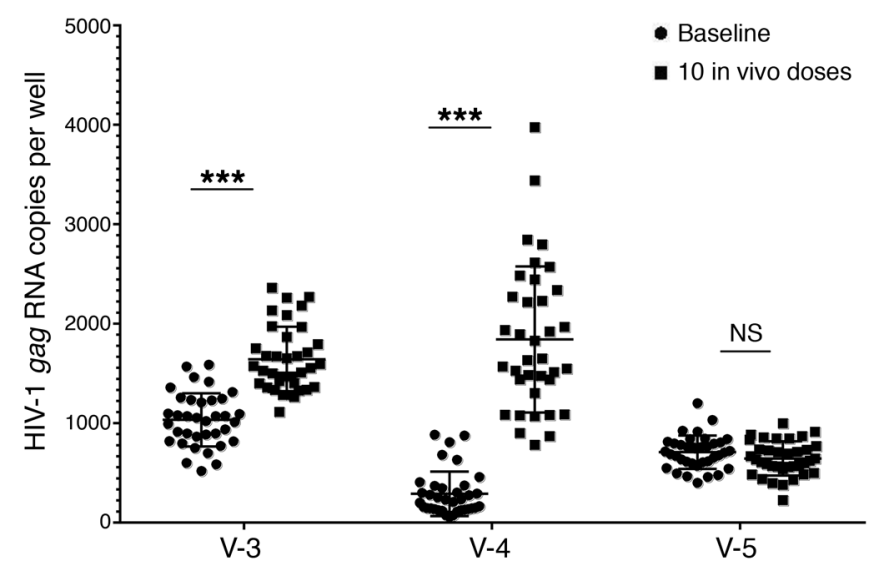

Figure 6. Multiple ( 10 doses) of in vivo VOR administered every 72 hours resulted in a sustained increase in HIV RNA in 2 of 3 participants. Data represent the mean $\pm \mathrm{SD}$. ${ }^{* *} P<0.0001$, by Mann-Whitney $U$ test.

immune function following this durable exposure to VOR failed to show any sign of reduced proliferation of HIV and non-HIV antigens or a reduced antiviral immune response. Nevertheless, the reversal of latency over this period did not lead to a depletion of persistent HIV infection in participants who maintained their ART. There may be several reasons why we did not observe a depletion of the reservoir. First, it is possible that the number of doses, even for patients who received 10 doses, was simply not enough to result in a measurable depletion of the reservoir. Second, the low frequency of latently infected cells in durably treated subjects may have caused insufficient chemokine or cytokine signaling by cells with reactivated virus, which could result in immune-mediated clearance. It is possible that there are latently infected cells in immune-privileged sites that, even if reactivated by VOR, are poorly accessed by immune cells and thus unable to affect virus clearance.

However, as some studies have suggested, depletion of the reservoir after latency reversal will most likely require the addition of an immune intervention to augment HIV-specific immunity. Use of a primary T cell model of HIV latency (24) showed that the cytopathic effect induced after latency reversal with VOR was insufficient to mediate cell death in the absence of global $\mathrm{T}$ cell activation. Furthermore, cytotoxic $\mathrm{T}$ lymphocytes (CTLs) from chronic, ART-treated patients with chronic HIV infection inefficiently cleared cells reactivated with VOR, while CTLs from elite controllers and CD8 ${ }^{+} \mathrm{T}$ cells prestimulated with Gag peptides effectively eliminated infected cells, presumably because of the high HIV-specific CTL activity of elite controllers and ex vivo Gag-stimulated $\mathrm{CD} 8^{+} \mathrm{T}$ cells. Thus, in many patients, the HIV-specific immune response may have waned in the absence of recent antigen exposure and/or may be dysfunctional or depleted by HIV disease. Furthermore, Deng et al. showed a widespread prevalence of CTL escape mutations in viral genomes found in the resting CD4 cell reservoir (25). Nevertheless, there were still some CTLs that recognized virus, and their activity could be enhanced by exogenous peptide stimulation. Although some individuals may respond to VOR given at shorter intervals, we are currently implementing studies combining immunotherapies with VOR administered at 72-hour
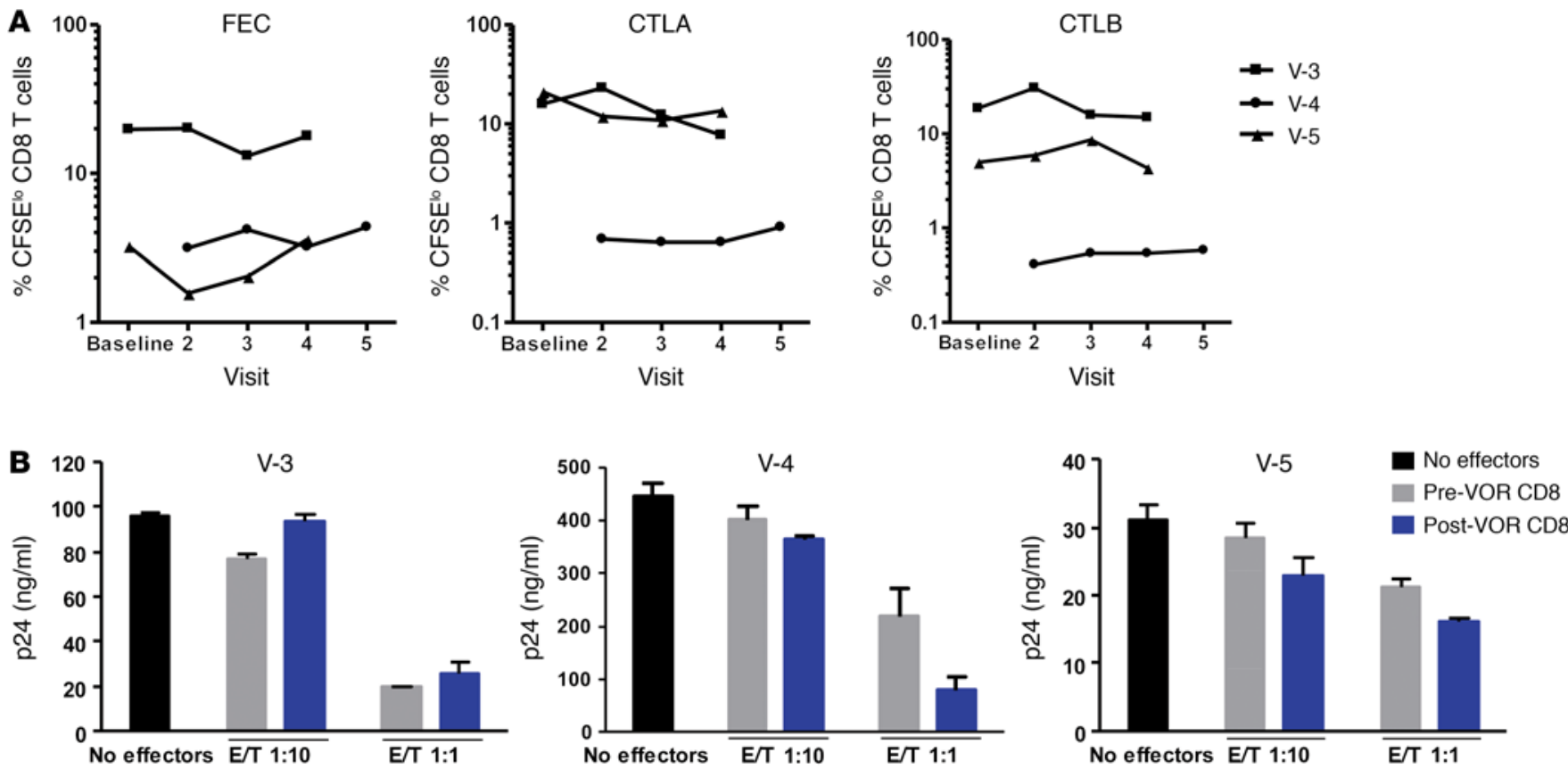

Figure 7. Repeated exposure to VOR (10 doses) had a limited effect on ex vivo $\mathrm{T}$ cell function. (A) Following in vivo exposure to VOR, CD8 ${ }^{+} \mathrm{T}$ cell proliferation in response to Flu peptide (FEC), HIV Gag/Nef peptide (CTLA), and other HIV peptides (CTLB) was evaluated by CFSE staining. Study clinic visits: 4-6 hours after a single VOR dose (visit 2); 4-6 hours after the second of paired VOR doses (visit 3); 4 hours and 7 months after the last of 10 VOR doses (visits 4 and 5, respectively). (B) In participants V-3, V-4, and V-5, in vivo exposure to VOR over a 1-month period did not reduce the antiviral capacity of CD8 ${ }^{+}$ $T$ cells when challenged with autologous targets infected with AR virus in a virus inhibition assay. 
intervals. Ultimately, studies to address whether LRAs such as VOR affect tissue reservoirs and whether cells containing reactivated virus can be cleared from immune-privileged sites will have to be included as part of future cure strategies.

We did not observe a statistically significant upregulation of rca-HIV RNA ex vivo in 4 of 16 participants, perhaps largely because baseline levels of rca-HIV RNA were 10 copies per well or fewer in 3 of 4 of these individuals (Supplemental Figure 1). In 5 of 12 participants in whom an ex vivo VOR response was measured, this response was not seen in vivo. There are numerous possible explanations for this, such as individual differences in VOR metabolism or distribution or a predominance of persistent replication-incompetent proviruses expressing measurable HIV RNA levels that mask HIV RNA induced from a smaller population of replication-competent proviruses (2628). It is also possible that some infected individuals carry a predominant population of latent virus that is less responsive to VOR, although the only study to address this question thus far found that HDAC inhibitors are broadly active across diverse viral integrants (29).

Measurements of rca-HIV RNA have limitations, and novel assays may improve the study of LRA activity in vivo. Increases in resting $\mathrm{CD}^{+}{ }^{+} \mathrm{T}$ cell-associated HIV gag p 24 antigen and in circulating p24 antigen levels after LRA exposure in vivo have been observed using an ultrasensitive assay recently described by Howell et al. (30). Further studies will be required to assess the ability to measure low levels of protein expression and compare rca-HIV RNA measures of latency reversal with antigen-based measures of latency reversal.

Although other LRAs are now under study, HDAC inhibitors are currently a leading potential therapeutic to attack latent HIV infection. It is reasonable to hypothesize that the use of combination therapies to efficiently disrupt latent infection and the use of vaccines or immunotherapies to assist in the clearance of latent HIV infection will be required to deplete latent, persistent HIV infection (31). A recent study combined the administration of the HDAC inhibitor romidepsin with an HIV vaccine (32), but robust depletion of persistent HIV infection was not noted. However, a full understanding of the optimal dosing schedule for romidepsin and its interaction with immunotherapies is still lacking. As we have previously shown for both CD8 and NK cell function in earlier VOR studies $(19,33)$, in this study, we observed no significant effect of VOR on CD8 immune function after repeated dosing in vivo.

Careful study of the effects of the kinetics of latency reversal and the interactions of LRAs with the antiviral immune response is required to advance toward the ultimate goal of developing therapies that can cure HIV infection. Additionally, understanding the kinetics of the effects of these compounds on host cell function may provide insight into successful clinical study design. Here, we have provided guidance to those wishing to use VOR as a tool in studies of HIV persistence and suggest that the serial administration of $400 \mathrm{mg}$ of the drug every 72 hours for up to 1 month is likely to be safe and well tolerated. We highlight the fact that latency reversal activity is demonstrable in the majority of, but not all, participants. However, if effective viral clearance strategies can be added in the future, evidence of
LRA activity that is not measured with our current assays may become apparent. Higher doses of VOR, more frequent dosing, or VOR administration over a period of more than 4 weeks might be considered, but we suspect that such strategies will incur an increased frequency of modest clinical toxicities. We view VOR as an important tool for research protocols, but improved LRAs are clearly needed. Nevertheless, our findings show that VOR can provide durable LRA activity, with minimal effects on the immune response.

\section{Methods}

Clinical protocol. In this phase I-II single-center study, HIV-infected participants were recruited if they were receiving stable standard-ofcare ART, with plasma HIV-1 RNA levels consistently below 50 copies/ $\mathrm{ml}$ and a CD4 count of more than 300/ $\mu$ l for at least 6 months prior to enrollment. Baseline ART was maintained throughout the study. All participants were men, $81 \%$ of whom were white, $19 \%$ black, and $6 \%$ Hispanic. The median age was 49 [range 26-66] years. At the time of study enrollment, all participants were virally suppressed $(<50$ copies/ml) for at least 0.7 years (0.7-9.2), with a median CD4 count of 718 (range, 402-1,302) and a median pre-ART CD4 nadir of 403 (range, 49-879) at the time of study enrollment (Table 1).

Measurement of cell-associated HIV RNA and DNA. The in vivo response to VOR (donated by Merck Research Laboratories) was measured as previously described, with minor modifications (4). When rca-HIV RNA assays were to be performed, the time of early morning VOR dosing was carefully standardized, such that cells would be uniformly harvested via apheresis 6 hours later to allow for adequate exposure to peak plasma VOR concentrations. Immediately following leukapheresis, resting $\mathrm{CD} 4^{+} \mathrm{T}$ cells were isolated as reported previously (2) and plated at 1 million cells/well, pelleted, snap-frozen, and stored at $-80^{\circ} \mathrm{C}$. For baseline samples, multiple, duplicate cell pellets were frozen and stored, so that at each study analysis time point, an untouched but identical baseline sample could be processed in parallel and rca-HIV RNA recovered, amplified, and compared with the rca-HIV RNA recovered from a sample of cells exposed to VOR in vivo that had been handled and processed simultaneously. Total RNA was isolated from 36 replicates of 1 million resting cells using the Magmax 96 Total RNA Isolation Kit (Life Technologies, Thermo Fisher Scientific) following the manufacturer's protocol. cDNA was synthesized in duplicate from DNase-treated, isolated RNA using the SuperScript III First-Strand Synthesis SuperMix Kit (Invitrogen, Thermo Fisher Scientific) according to the manufacturer's protocol. Reverse transcriptase was omitted from duplicate wells of each treatment condition, and those wells served as controls for DNA contamination. Duplicate cDNAs were pooled, and triplicate PCR amplifications were performed using the Bio-Rad CFX96 Real-Time PCR System and previously published primers and probes (34). A standard curve was generated for each PCR reaction as described previously (4). The results of the PCR replicates representing each of the original 36 pools of RNA were averaged, and the SD was determined for each condition. The Mann-Whitney $U$ test was used to calculate the statistical significance of all comparisons.

To measure total cell-associated HIV DNA, 5 million peripheral blood mononuclear cells (PBMCs) and 5 million purified resting $\mathrm{CD}^{+} \mathrm{T}$ cells from each time point were snap-frozen and stored at 
$-80^{\circ} \mathrm{C}$. DNA was extracted from cells using the DNeasy Blood and Tissue Kit (QIAGEN) following the manufacturer's instructions. Amplification of HIV gag DNA was performed in triplicate on a Bio-Rad CFX96 Real-Time PCR System using previously published primers and probes, and an HIV-1 gag standard was generated as previously reported $(8,34,35)$.

Quantitative viral outgrowth assay. Lymphocytes were obtained by continuous-flow leukapheresis. Isolation of resting $\mathrm{CD}^{+} \mathrm{T}$ cells and recovery and quantification of replication-competent virus were performed as described previously (36). Briefly, approximately 34 to 49 million resting $\mathrm{CD}^{+} \mathrm{T}$ cells were plated in replicate-limiting dilutions of 2.5 million (12-18 cultures), 0.5 million ( 6 cultures), and 0.1 million ( 6 cultures) cells per well and were activated with phytohemagglutinin (PHA) (Remel Products, Thermo Fisher Scientific), a 5-fold excess of allogeneic irradiated PBMCs from a seronegative donor, and $60 \mathrm{U} / \mathrm{ml} \mathrm{IL-2}$ for 24 hours. Cultures were washed and cocultivated with CD8-depleted PBMCs collected from selected HIV-seronegative donors screened for adequate CCR5 expression. Culture supernatants were harvested on days 15 and 19 and assayed for virus production by HIV p24 antigen capture ELISA (ABL Inc.). Cultures were scored as positive if p24 was detected on day 15 and was increased in concentration on day 19. The number of resting $\mathrm{CD}^{+} \mathrm{T}$ cells in infected units per billion (IUPB) was estimated by a maximum likelihood method $(37,38)$.

Peptides. Peptides were synthesized by Sigma-Genosys. HIV-1 $\mathrm{CD}^{+} \mathrm{T}$ cell optimal epitopes (www.hiv.lanl.gov/content/sequence/ ELF/epitope_analyzer.html) were grouped into pools by protein (either Gag/Nef or non-Gag/NEF HIV-1 proteins). In selected donors, responses to a pool of influenza, EBV, and $\mathrm{CMV} \mathrm{CD8}{ }^{+} \mathrm{T}$ cell epitopes (39) were also quantified.

$T$ cell proliferation assay. Cryopreserved PBMCs were thawed and rested at $37^{\circ} \mathrm{C}$ overnight in $\mathrm{R} 10$, and then pulsed under rotation with $5 \mu \mathrm{M}$ CFSE for 10 minutes. The reaction was quenched with ice-cold R10, and excess CFSE was removed by washing twice with PBS containing 5\% FBS. PBMCs were then incubated with $2 \mu \mathrm{g} /$ $\mathrm{ml}$ peptide plus 10 units $/ \mathrm{ml} \mathrm{IL-2}$ at $37^{\circ} \mathrm{C}$ for 5 days. R10 with $0.5 \%$ DMSO plus 10 units/ml IL-2 was the negative control, and $3 \mu \mathrm{g} / \mathrm{ml}$ PHA plus 10 units/ml IL-2 was the positive control. After 5 days, the PBMCs were stained with Zombie NIR Viability Dye, followed by CD3-Brilliant Violet 421, CD4-Brilliant Violet 650, CD8-Brilliant Violet 510, and CD14/16/19/56-PerCP (dump) (all from BioLegend) and then acquired using a BD LSR II Flow Cytometer. Data were analyzed using FlowJo, version 10, and ModFitLT, version 4 (Verity Software House).

Viral inhibition assay. A unique autologous viral inhibition assay was performed as previously described $(1,2)$. Autologous CD8 ${ }^{+} \mathrm{T}$ cells were isolated from PBMCs by positive selection (EasySep Human $\mathrm{CD}^{+}$Selection Kit; STEMCELL Technologies). Autologous CD8-depleted PBMCs were first activated with $4 \mu \mathrm{g} / \mathrm{ml}$ PHA (Remel) and $60 \mathrm{U} / \mathrm{ml} \mathrm{IL-2}$ and then infected by spinoculation at 1,200 $g$ for 90 minutes with either HIV-1 strain JR-CSF or autologous reservoir (AR) virus at an MOI of 0.01. AR virus was obtained from pooled supernatants of replicate wells from outgrowth assays of resting $\mathrm{CD} 4^{+} \mathrm{T}$ cells for each patient. Fifty thousand $\left(5 \times 10^{4}\right)$ targets/well were cocultured with $\mathrm{CD}^{+} \mathrm{T}$ cells in triplicate at the indicated (Figure 7B) E/T ratio in $0.2 \mathrm{ml}$ conditioned Iscove's modified Dulbecco's medium (cIMDM) supplemented with 10\% FBS,
$1 \%$ penicillin-streptomycin, and $5 \mathrm{U} / \mathrm{ml} \mathrm{IL-2}$. The p24 concentration in supernatant was assayed by p24 ELISA (ABL Inc.) on day 6.

Statistics. Statistical comparisons between pre- and post-VOR exposure groups were analyzed with a Mann-Whitney $U$ test using Graph Pad Prism 7.0 software (GraphPad Software). P values of less than 0.05 were considered significant. Pools of 36 million resting $\mathrm{CD}^{+} \mathrm{T}$ cells from each time point were analyzed for Figures 4-6, and as described in the legends for other figures. The mean \pm SD is reported. For virus inhibition assays, statistical comparisons between groups were analyzed by 1-way ANOVA with Dunnett's post-test correction for multiple comparisons using GraphPad Prism.

Study approval. This study was reviewed and approved by the IRB of the University of North Carolina, the National Institute of Allergy and Infectious Diseases (NIAID) Clinical Sciences Review Committee, and the FDA. This study is registered at Clinicaltrials. gov, and clinical trial monitoring was provided by the NIAID. All participants provided informed consent prior to their enrollment in the study.

\section{Author contributions}

DMM, NMA, JE, and CLG designed the study. DMM, CLG, JE, and JDK provided clinical management and study coordination. JLK, NMA, BA, KS, and ES measured rca-HIV RNA, performed QVOA, and analyzed the data. NG, JAMS, GC, and YX designed and performed $\mathrm{CD}^{+} \mathrm{T}$ cell function experiments. ADK oversaw pharmacokinetic measurements. NMA and DMM reviewed the data and wrote the manuscript. All authors discussed the results and commented on the manuscript.

\section{Acknowledgments}

This study was supported by NIH grants U01 AI095052 (to DMM); RR024383 (to the UNC TraCS Institute); AI50410 (to the UNC Center for AIDS Research); and (P30 CA016086, to support in part the UNC Flow Cytometry Core); and by a grant from the National Center for Advancing Translational Sciences (KL2 TR001109, to JMS). We thank D. Hazuda, R. Barnard, B. Howell, and S. Lehrman (Merck Research Laboratories, West Point, PA, USA) for study advice and pharmaceutical assistance; R. Bosch (Harvard School of Public Health, Boston, MA, USA) and M. Hudgens (UNC Chapel Hill, Chapel Hill, NC, USA) for statistical analysis advice; R. Bedimo (UT Southwestern, Dallas, TX, USA), W.C. Miller (The Ohio State University, Columbus, OH, USA), M. Floris-Moore, and C. van der Horst (UNC Chapel Hill, Chapel Hill, NC, USA) for data safety monitoring review; and at UNC Chapel Hill, M. Cottrell for pharmacological assays; the UNC CFAR Virology and Immunology Core; J.P. Murphy for technical support; A. Crooks, D. Chen, V. Candler, and B. Turner for study and data coordination; and Y. Park and the UNC Blood Bank. Finally, we are grateful for the contributions of the participants, whose altruism makes these studies possible.

Address correspondence to: Nancie M. Archin or David M. Margolis, UNC HIV Cure Center, UNC Chapel Hill, 120 Mason Farm Road, CB 7042, Chapel Hill, North Carolina 27599-7042, USA. Phone: 919.962.0180; Email: nancie_archin@med.unc.edu (N.M. Archin). Phone: 919.966.6388; Email: dmargo@med.unc.edu (D.M. Margolis). 
1. Glozak MA, Seto E. Histone deacetylases and cancer. Oncogene. 2007;26(37):5420-5432.

2. Keedy KS, Archin NM, Gates AT, Espeseth A, Hazuda DJ, Margolis DM. A limited group of class I histone deacetylases acts to repress human immunodeficiency virus type 1 expression. J Virol. 2009;83(10):4749-4756.

3. Rasmussen TA, Lewin SR. Shocking HIV out of hiding: where are we with clinical trials of latency reversing agents? Curr Opin HIV AIDS. 2016;11(4):394-401.

4. Archin NM, et al. Administration of vorinostat disrupts HIV-1 latency in patients on antiretroviral therapy. Nature. 2012;487(7408):482-485.

5. Elliott JH, et al. Activation of HIV transcription with short-course vorinostat in HIV-infected patients on suppressive antiretroviral therapy. PLoS Pathog. 2014;10(10):e1004473.

6. Rasmussen TA, et al. Panobinostat, a histone deacetylase inhibitor, for latent-virus reactivation in HIV-infected patients on suppressive antiretroviral therapy: a phase $1 / 2$, single group, clinical trial. Lancet HIV. 2014;1(1):e13-e21.

7. Søgaard OS, et al. The depsipeptide romidepsin reverses HIV-1 latency in vivo. PLoS Pathog. 2015;11(9):e1005142.

8. Archin NM, et al. HIV-1 expression within resting $\mathrm{CD}^{+}{ }^{+} \mathrm{T}$ cells after multiple doses of vorinostat. JInfect Dis. 2014;210(5):728-735.

9. Kuo-Hsiung Y, et al. HIV-1 expression within resting CD4 T-cells following multiple doses of vorinostat in vivo. In: Conference on Retroviruses and Opportunistic Infections; March 3-6, 2014; Boston, MA. Abstract 435LB

10. White $\mathrm{CH}$, et al. Mixed effects of suberoylanilide hydroxamic acid (SAHA) on the host transcriptome and proteome and their implications for HIV reactivation from latency. Antiviral Res. 2015;123:78-85.

11. Reardon B, et al. Dose-responsive gene expression in suberoylanilide hydroxamic acid-treated resting CD4 ${ }^{+}$T cells. AIDS. 2015;29(17):2235-2244.

12. Halsall JA, Turan N, Wiersma M, Turner BM. Cells adapt to the epigenomic disruption caused by histone deacetylase inhibitors through a coordinated, chromatin-mediated transcriptional response. Epigenetics Chromatin. 2015;8:29.

13. Archin NM, Sung JM, Garrido C, Soriano-Sarabia N, Margolis DM. Eradicating HIV-1 infection: seeking to clear a persistent pathogen. Nat Rev
Microbiol. 2014;12(11):750-764.

14. Bruner KM, Hosmane NN, Siliciano RF. Towards an HIV-1 cure: measuring the latent reservoir. Trends Microbiol. 2015;23(4):192-203.

15. Li JZ, et al. The size of the expressed HIV reservoir predicts timing of viral rebound after treatment interruption. AIDS. 2016;30(3):343-353.

16. Gutiérrez C, et al. Bryostatin-1 for latent virus reactivation in HIV-infected patients on antiretroviral therapy. AIDS. 2016;30(9):1385-1392.

17. Elliott JH, et al. Short-term administration of disulfiram for reversal of latent HIV infection: a phase 2 dose-escalation study. Lancet HIV. 2015;2(12):e520-e529.

18. Sung JA, et al. Dual-Affinity Re-Targeting proteins direct $\mathrm{T}$ cell-mediated cytolysis of latently HIV-infected cells. JClin Invest. 2015;125(11):4077-4090.

19. Sung JA, et al. Expanded cytotoxic T-cell lymphocytes target the latent HIV reservoir. J Infect Dis. 2015;212(2):258-263.

20. Beliakova-Bethell N, et al. Suberoylanilide hydroxamic acid induces limited changes in the transcriptome of primary CD4(+) T cells. AIDS. 2013;27(1):29-37.

21. Bartholomeeusen K, Fujinaga K, Xiang Y, Peterlin BM. Histone deacetylase inhibitors (HDACis) that release the positive transcription elongation factor $\mathrm{b}$ (P-TEFb) from its inhibitory complex also activate HIV transcription. J Biol Chem. 2013;288(20):14400-14407.

22. Ke R, Lewin SR, Elliott JH, Perelson AS. Modeling the effects of vorinostat in vivo reveals both transient and delayed HIV transcriptional activation and minimal killing of latently infected cells. PLoS Pathog. 2015;11(10):e1005237.

23. Peart MJ, et al. Identification and functional significance of genes regulated by structurally different histone deacetylase inhibitors. Proc Natl Acad Sci U S A. 2005;102(10):3697-3702.

24. Shan L, et al. Stimulation of HIV-1-specific cytolytic T lymphocytes facilitates elimination of latent viral reservoir after virus reactivation. Immunity. 2012;36(3):491-501.

25. Deng K, et al. Broad CTL response is required to clear latent HIV-1 due to dominance of escape mutations. Nature. 2015;517(7534):381-385.

26. Ho YC, et al. Replication-competent noninduced proviruses in the latent reservoir increase barrier to HIV-1 cure. Cell. 2013;155(3):540-551.

27. Simonetti FR, et al. Clonally expanded CD $4^{+}$ $\mathrm{T}$ cells can produce infectious HIV- 1 in vivo. Proc Natl Acad Sci U S A. 2016;113(7):1883-1888.

28. Lorenzi JC, et al. Paired quantitative and qualitative assessment of the replication-competent HIV-1 reservoir and comparison with integrated proviral DNA. Proc Natl Acad Sci U S A. 2016;113(49):E7908-E7916.

29. Barton K, et al. Broad activation of latent HIV-1 in vivo. Nat Commun. 2016;7:12731.

30. Howell B. Ultrasensitive detection of viral p24 following reactivation of latent HIV. Poster presented at: Keystone Symposium: HIV Persistence: Pathogenesis and Eradication; March 20-24, 2016; Olympic Valley, California.

31. Margolis DM, Garcia JV, Hazuda DJ, Haynes BF. Latency reversal and viral clearance to cure HIV1. Science. 2016;353(6297):aaf6517.

32. Leth $\mathrm{S}$, et al. Combined effect of Vacc-4x, recombinant human granulocyte macrophage colony-stimulating factor vaccination, and romidepsin on the HIV-1 reservoir (REDUC): a single-arm, phase 1B/2A trial. Lancet HIV. 2016;3(10):e463-e472.

33. Garrido C, et al. HIV latency-reversing agents have diverse effects on natural killer cell function. Front Immunol. 2016;7:356.

34. Israel-Ballard K, et al. TaqMan RT-PCR and VERSANT HIV-1 RNA 3.0 (bDNA) assay Quantification of HIV-1 RNA viral load in breast milk. JClin Virol. 2005;34(4):253-256.

35. Robinson LH, Gale CV, Kleim JP. Inclusion of full length human immunodeficiency virus type 1 (HIV-1) gag sequences in viral recombinants applied to drug susceptibility phenotyping. J Virol Methods. 2002;104(2):147-160.

36. Archin NM, et al. Valproic acid without intensified antiviral therapy has limited impact on persistent HIV infection of resting $\mathrm{CD} 4^{+} \mathrm{T}$ cells. AIDS. 2008;22(10):1131-1135.

37. Macken C. Design and analysis of serial limiting dilution assays with small sample sizes. J Immunol Methods. 1999;222(1-2):13-29.

38. Myers LE, McQuay LJ, Hollinger FB. Dilution assay statistics. JClin Microbiol. 1994;32(3):732-739.

39. Currier JR, et al. A panel of MHC class I restricted viral peptides for use as a quality control for vaccine trial ELISPOT assays. J Immunol Methods. 2002;260(1-2):157-172. 